\title{
Solid lipid nanoparticles modified with stearic acid-octaarginine for oral administration of insulin
}

This article was published in the following Dove Press journal:

International Journal of Nanomedicine

2 July 2012

Number of times this article has been viewed

\author{
Zhen-Hai Zhang ${ }^{1,2}$ \\ Yin-Long Zhang ${ }^{2}$ \\ Jian-Ping Zhou ${ }^{2}$ \\ Hui-Xia Lv ${ }^{2}$ \\ 'Jiangsu Province Academy of \\ Traditional Chinese Medicine, Nanjing, \\ China; ${ }^{2}$ Department of Pharmaceutics, \\ China Pharmaceutical University, \\ Nanjing, China
}

\begin{abstract}
The aim of this study was to design and characterize solid lipid nanoparticles (SLNs) modified with stearic acid-octaarginine $\left(\mathrm{SA}-\mathrm{R}_{8}\right)$ as carriers for oral administration of insulin (SA$\mathrm{R}_{8}$-Ins-SLNs). The SLNs were prepared by spontaneous emulsion solvent diffusion methods. The mean particle size, zeta potential, drug loading, and encapsulation efficiency of the SA-R $\mathrm{R}_{8}$-InsSLNs were $162 \mathrm{~nm}, 29.87 \mathrm{mV}, 3.19 \%$, and $76.54 \%$, respectively. The zeta potential of the SLNs changed dramatically, from $-32.13 \mathrm{mV}$ to $29.87 \mathrm{mV}$, by binding the positively charged SA- $\mathrm{R}_{8}$. Morphological studies of SA-R $\mathrm{R}_{8}$-Ins-SLNs using transmission electron microscopy showed that they were spherical. In vitro, a degradation experiment by enzymes showed that SLNs and $\mathrm{SA}-\mathrm{R}_{8}$ could partially protect insulin from proteolysis. Compared to the insulin solution,

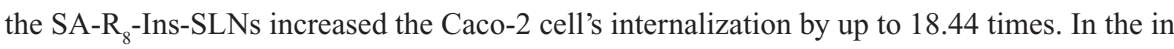
vivo studies, a significant hypoglycemic effect in diabetic rats over controls was obtained, with a SA-R - Ins-SLN pharmacological availability value of $13.86 \pm 0.79$. These results demonstrate that $\mathrm{SA}-\mathrm{R}_{8}$-modified SLNs promote the oral absorption of insulin.
\end{abstract}

Keywords: solid lipid nanoparticles, cell penetration peptides, stearic acid octaarginine, insulin, oral administration

\section{Introduction}

Oral delivery of insulin as a noninvasive therapy for diabetes mellitus is still a challenge to drug-delivery technology, as it cannot be administered currently via the oral route, due to rapid enzymatic degradation in the stomach, inactivation and digestion by enzymes in the intestinal lumen, and poor permeability across the intestinal epithelium, due to its high molecular weight and lack of lipophilicity. ${ }^{1}$ However, oral insulin is advantageous because it is delivered to the liver, its primary site of action, via portal circulation, a mechanism very similar to endogenous insulin. ${ }^{2}$

Different formulation approaches have been investigated to overcome the gastrointestinal tract barriers for the delivery of insulin via the oral route, such as the use of liposomes, microemulsion, microspheres, and nanoparticles. ${ }^{3}$ Among the possible strategies for oral delivery of insulin, solid lipid nanoparticles (SLNs) represent a promising approach. ${ }^{4}$ The advantages of SLNs include improved efficacy, reduced toxicity, protection of active compounds, and enhanced biocompatibility. SLNs have a large specific surface area of physiological compatible lipid matrix, and their protection power against the gastrointestinal environment is mostly dependent on the degree of protein encapsulation within these nanostructures. ${ }^{5,6}$ Furthermore, the carrier itself can be taken up, to a certain extent, by epithelial cells or the lymphoid tissues in Peyer's patches. ${ }^{7}$ 
Cell-penetrating peptides have attracted increasing attention in recent years as a promising vehicle for facilitating the cellular uptake of various molecular cargos. ${ }^{8}$ Among them, arginine-rich derivatives of the HIV Tat are the most widely studied peptides. ${ }^{9-11}$ It is well know that homopeptides consisting of 7-9 arginines display higher cellular uptake compared to the Tat peptide itself. ${ }^{12}$ Kamei et al proved that cell-penetrating peptides could improve the intestinal absorption of insulin. ${ }^{13}$ Furthermore, the addition of fatty acid to polyarginines dramatically improves their cellular uptake. ${ }^{14-16}$

Therefore, in the present study, insulin SLNs modified with stearic acid-octaarginine (SA- $\mathrm{R}_{8}$-Ins-SLNs) were prepared in order to improve the stability and bioavailability of orally administered insulin. The spontaneous emulsion solvent diffusion method was adopted, and the formed SLNs were then characterized and evaluated by dynamic light scattering, high-performance liquid chromatography (HPLC) assay, transmission electron microscopy (TEM), internalization effect in Caco-2 cells, and hypoglycemic experiment in diabetic rats.

\section{Materials and methods Materials}

Insulin (27.5 IU/mg) was purchased from Xuzhou Wanbang Biochemical Pharmaceutical Co, Ltd (Xuzhou, China). Stearic acid-octaarginine (SA-R ${ }_{8}$ ) was purchased from Shanghai Gil Chemical Co, Ltd (Shanghai, China). Soybean phospholipid was obtained from Shanghai Taiwei Pharmaceutical Co, Ltd (Shanghai, China). Monostearin, poloxamer 188, and polysorbate 80 were purchased from Shanghai Chemical Reagent Co, Ltd (Shanghai, China). 3-(4,5-dimethylthiazol2-yl)-2,5-diphenyl-tetrazolium bromide (MTT) was obtained from Sigma (St Louis, MO). Trypsin and Dulbecco's modified Eagle's medium were purchased from Gibco BRL (Gaithersburg, MD). All other solvents were of analytical or chromatographic grades and commercially available.

\section{Cell culture and animal models}

The Caco-2 cells, a kind gift from Dr Moniqué Rousset of INSERM U178 (Villejuif, France), were cultured in Dulbecco's modified Eagle's medium containing 10\% fetal calf serum. Sprague Dawley diabetic rats, weighing $200 \pm 10 \mathrm{~g}$, were obtained from the Animal Center of China Pharmaceutical University, and all the animal experiments were performed according to the Guiding Principles of the Care and Use of Experiment Animals at China Pharmaceutical University. The study protocol was reviewed and approved by Institutional Animal Care and Use Committee, China Pharmaceutical University, China.

\section{The preparation of SLNs}

The SLNs, loaded with insulin and $S A-R_{8}\left(S A-R_{8}\right.$-InsSLNs), were prepared using the spontaneous emulsion solvent diffusion method: $5 \mathrm{mg}$ of insulin were dissolved in $2 \mathrm{~mL} 0.01 \mathrm{M}$ hydrochloric acid (inner aqueous phase) and added to a $10 \mathrm{~mL}$ acetone solution containing $90 \mathrm{mg}$ of stearic acid and $10 \mathrm{mg}$ of soybean phospholipids (oily phase). The resulting mixtures were dispersed at $50 \mathrm{~W}$ for 15 seconds with an ultrasonic probe (JY92-II ultrasonic processor; Ningbo Scientz Biotechnology Co, Ltd, Ningbo, China) leading to $\mathrm{W} / \mathrm{O}$ emulsions. Then, the emulsions were poured into $100 \mathrm{~mL} 1.6 \%$ poloxamer 188 and $15 \% \mathrm{SA}-\mathrm{R}_{8}$ solution under continuous stirring, using a DC-40 stirrer (Hangzhou Electrical Engineering Instruments, Hangzhou, China) at $500 \mathrm{rpm}$ for 1 hour, in order to form SLNs. The untrapped drug and adjuvant were removed by filtration. The preformed SLNs were filtered through a $0.45 \mu \mathrm{m}$ cellulose nitrate filter membrane, and then lyophilized in the presence of $5 \%$ mannitol. The lyophilized powders were kept in a refrigerator at $4{ }^{\circ} \mathrm{C}$. SLNs containing insulin without $\mathrm{SA}_{8}$ (Ins-SLNs) were also prepared in the same manner to serve as controls. All experiments were performed in triplicate.

\section{The characterization of SLNs}

Determination of the encapsulation efficiency and drug loading of the insulin

Insulin entrapment efficiency (EE) and drug loading (DL) of the SLNs were determined by HPLC, as reported previously (see equations (1) and (2), respectively). ${ }^{18}$

$$
\begin{aligned}
& \mathrm{EE}(\%)=\frac{\text { Weight of insulin in SLNs }}{\text { Weight of insulin fed initially }} \times 100 \\
& \mathrm{DL}(\%)=\frac{\text { Weight of insulin in SLNs }}{\text { Weight of SLNs }} \times 100
\end{aligned}
$$

\section{Determination of the DL and binding efficiency of $S A-R_{8}$}

The SA- $\mathrm{R}_{8}$-Ins-SLNs were dissolved in 50\% ethanol under water bath at $70^{\circ} \mathrm{C}$ for 10 minutes, and then cooled to room temperature to preferentially precipitate the lipid. The SA- $\mathrm{R}_{8}$ content in the supernatant, after centrifugation $\left(14,000 \mathrm{rpm}\right.$ for 10 minutes at $4^{\circ} \mathrm{C}$, L8-60M; Beckman, 
Fullerton, CA) in an ultimate filtration tube (molecular weight cutoff $=10,000$ ), was measured by an HPLC method at $220 \mathrm{~nm}$ using an LC-10 Avp pump and an LC-10 Avp UVVIS detector (both obtained from Shimadzu, Kyoto, Japan). The chromatographic conditions employed were as follows: mixture of acetonitrile and water $(51: 49, \mathrm{v} / \mathrm{v})$ as a mobile phase, Shim-pack CLC-ODS column at a temperature of $30^{\circ} \mathrm{C}(5 \mu \mathrm{m}, 150 \mathrm{~mm}, 6 \mathrm{~mm}$ id $)$, flow rate of $1.0 \mathrm{~mL} \cdot \mathrm{min}^{-1}$, and sample injection volume of $20 \mu \mathrm{L}$.

The calibration curve for the quantification of $S A-\mathrm{R}_{8}$ was linear over a range of standard concentrations of SA- $\mathrm{R}_{8}$, from $10 \mu \mathrm{g} \cdot \mathrm{mL}^{-1}$ to $200 \mu \mathrm{g} \cdot \mathrm{mL}^{-1}$, with a correlation coefficient of $R^{2}=0.9997$. The limit of detection was $10 \mu \mathrm{g} \cdot \mathrm{mL}^{-1}$. The binding efficiency (BE) and DL of SA-R $\mathrm{R}_{8}$ attached to the SLNs were calculated using equations (3) and (4), respectively:

$$
\begin{gathered}
\mathrm{BE} \%=\frac{\text { Weight of SA- } \mathrm{R}_{8} \text { in SLNs }}{\text { Weight of SA-R } \mathrm{R}_{8} \text { fed initially }} \times 100 \% \\
\mathrm{DL} \%=\frac{\text { Weight of SA- } \mathrm{R}_{8} \text { in SLNs }}{\text { Weight of SLNs }} \times 100 \%
\end{gathered}
$$

\section{Particle size and zeta potential measurement}

The mean particle size and the zeta potential of the SA- $\mathrm{R}_{8}-$ Ins-SLNs were determined with a Zetasizer (3000 HS; Malvern Instruments, Malvern, UK). Its morphology was characterized by TEM (NanoScope V; Veeco/Digital Instruments, Plainview, NY), with a $5 \mu \mathrm{m}$ scanner in contact mode.

\section{Enzymatic stability studies with pepsin and trypsin}

To assess the protective effect against gastrointestinal degradation, the $\mathrm{SA}-\mathrm{R}_{8}$-Ins-SLNs were incubated with a simulated gastric fluid $\mathrm{pH}=1.2$ (with pepsin) and a simulated intestinal fluid $\mathrm{pH}=6.8$ (with trypsin) in a water bath oscillator at $37^{\circ} \mathrm{C}$. At predetermined time intervals, samples were withdrawn and the enzymatic reaction stopped by $0.05 \mathrm{~mol} / \mathrm{L} \mathrm{NaOH}$ (pepsin) and $0.1 \mathrm{~mol} / \mathrm{L} \mathrm{HCL}$ (trypsin). The remaining amount of insulin was assayed with HPLC. All the experiments were performed in hexaplicate.

\section{Transport experiments in the Caco-2 cell}

Caco-2 cell monolayers were prepared by cultivating Caco- 2 cells on polycarbonate membrane filters for 3 weeks. The Caco- 2 cells were seeded on 6 -well plates at $2 \times 10^{5}$ cells per well, and then incubated for 24 hours. The culture medium was replaced by the uptake medium. ${ }^{17}$ For the uptake studies, insulin samples were used in free form and in the form of
SLNs. Then, $2.0 \mathrm{~mL}$ of $0.25 \mathrm{mg} / \mathrm{mL}$ final concentration of insulin, Ins-SLNs, and SA-R -Ins-SLNs were added to the apical side of the cell culture dish. The cells were incubated at $37^{\circ} \mathrm{C}$, and $200 \mu \mathrm{L}$ samples were withdrawn from the basolateral part at the predetermined times of $15,30,45,60$, and 120 minutes, and replaced with equal volumes of fresh HEPES-buffered Hank's balanced salt solution. The samples were then analyzed for insulin content by the HPLC method. All the experiments were conducted in triplicate.

Apparent permeability coefficients $\left(P_{\text {app }}\right)$ were calculated according to the following equation:

$$
P_{\text {app }}=(d Q / d t)\left(C_{0} * 60 / A\right)
$$

where $P_{\text {app }}$ is the apparent permeability in $\mathrm{cm} / \mathrm{s}, d Q / d t$ is the permeability rate, $\mathrm{A}$ is the diffusion area of the monolayer $\left(\mathrm{cm}^{2}\right)$, and $C_{0}$ is the initial concentration of the insulin. Statistical differences were calculated by using one-way analyses of variance at a significant level of $P<0.05$.

Absorption enhancement ratio $(R)$ was calculated as follows:

$$
R=P_{\text {app (sample })} / P_{\text {app (control })}
$$

\section{In vivo hypoglycemic effect}

The lyophilized powder of Ins-SLNs with and without $\mathrm{SA}-\mathrm{R}_{8}$ was dissolved in distilled water and administered to the duodenum of fasted diabetic rats, at an insulin dose of $25 \mathrm{IU} / \mathrm{kg}$. Insulin powder delivered orally at $25 \mathrm{IU} / \mathrm{kg}$ and subcutaneous injection at $2 \mathrm{IU} / \mathrm{kg}$ served as controls. Each group contained six rats. Blood samples were collected from the tail vein before drug administration and at $0.5,1,2,3,5$, 8,10 , and 12 hours after duodenal drug administration, and blood glucose levels were determined by using a glucose GOD-PAD kit (Shanghai Kexin Biochemical Reagent Industry, Shanghai, China).

PA\% was calculated by using the following equations:

$$
\mathrm{PA} \%=\frac{A A C_{\text {duodenal }} \times \text { Dose }_{S C}}{A A C_{S C} \times \text { Dose }_{\text {duodenal }}} \times 100
$$

where $A A C$ refers to the areas above the glucose reductiontime curve, which was calculated from time 0 hours to 12 hours by using the trapezoid method. ${ }^{18}$

Statistical analysis was performed by using Student's $t$-test, and the differences were judged to be significant at $P<0.05$. 
Table I DL, EE, average diameter, PDI, and zeta potential of Ins-SLNs and SA-R $R_{8}$-Ins-SLNs

\begin{tabular}{|c|c|c|c|c|c|c|c|}
\hline Sample & DL\% of insulin & EE\% of insulin & DL\% of SA-R & EE\% of SA-R & Diameter (nm) & PDI & $\zeta *(\mathrm{mV})$ \\
\hline Ins-SLNs & $3.09 \pm 0.45$ & $64.89 \pm 3.55$ & - & - & $137.4 \pm 12.19$ & $0.21 \pm 0.07$ & $-32.13 \pm 1.05$ \\
\hline$S A-R_{8}-$ Ins-SLNs & $3.19 \pm 0.32$ & $76.54 \pm 2.18$ & $11.74 \pm 1.54$ & $93.92 \pm 3.32$ & $162.4 \pm 15.23$ & $0.14 \pm 0.02$ & $29.87 \pm 4.53$ \\
\hline
\end{tabular}

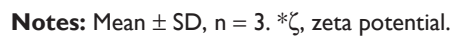

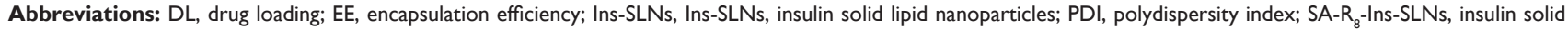
lipid nanoparticles modified with stearic acid-octaarginine; SD, standard deviation.

\section{Results and discussion The preparation of SLNs}

Preliminary studies were carried out to determine the relationship between the formulation parameters and the properties of the Ins-SLNs. Two major parameters affected the properties of the SLNs: the stearic acid:insulin weight ratio, which exerted a significant effect on the $\mathrm{EE} \%$ of insulin; and the $\mathrm{SA}-\mathrm{R}_{8}$ and soybean phospholipid concentration in the oily phase, which affected the size distribution of the Ins-SLNs. An increase in the relative amount of stearic acid to insulin resulted in an increase in the EE\% of insulin and SA- $R_{8}$. When the ratio reached 15:1, the highest EE\% of insulin and $\mathrm{SA}-\mathrm{R}_{8}$ was achieved, and an even higher ratio would no longer increase them. The appropriate percentage of soybean phospholipids was $5 \% \sim 10 \%$. Below $5 \%$ of the SLN matrix, the average diameter was larger than $200 \mathrm{~nm}$; above $10 \%$ of the SLNs matrix, the polydispersity index of the nanoparticles increased. Furthermore, the appropriate percentage of $\mathrm{SA}-\mathrm{R}_{8}$ in the formulation was limited to $10 \% \sim 15 \%$ of the SLN matrix. At this percentage, the zeta potential value of the $\mathrm{SA}-\mathrm{R}_{8}$-Ins-SLNs was $30 \mathrm{mV}$, below $8 \%$

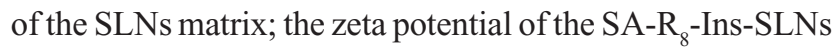
was lower than $20 \mathrm{mV}$, and a higher SA-R amount (above $15 \%$ ) did not increase its DL\% or zeta potential in the SLNs, but inversely lowered its EE\%. Other influential factors, such as the concentration of poloxamer 188, volume of inner aqueous phase, and volume of oily phase were investigated, and it was found that they contributed little to the properties of the SLNs. Thus, the optimal formulation of the Ins-SLNs was chosen as follows: $5 \mathrm{mg}$ insulin, $15 \mathrm{mg} \mathrm{SA}-\mathrm{R}_{8}, 90 \mathrm{mg}$ stearic acid, and $10 \mathrm{mg}$ soybean phospholipids as the oily phase, and poloxamer 188 (1.6\% solution) as the stable agent. For comparing with the SA-R - -Ins-SLNs, the Ins-SLNs were prepared using the same method used for SA-R - Ins-SLNs, but without $S A-R_{8}$. The characterization results of $S A-R_{8}-$ Ins-SLNs and Ins-SLNs are listed in Table 1.

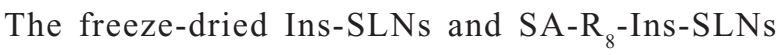
appeared as a white powder, and they were easily dispersed in water to form translucent solutions with a visible, sky-blue opalescence. As revealed by TEM (Figure 1), the SA-R -Ins-
SLNs exhibited a smooth, spherical morphology and were well dispersed and separated. Furthermore, the size of the SA-R-Ins-SLNs determined with TEM (about $120 \mathrm{~nm}$ ) was smaller than that of the SA- $\mathrm{R}_{8}$-Ins-SLNs in an aqueous phase ( $162.4 \mathrm{~nm} \pm 15.23 \mathrm{~nm}$ ), which might be caused by the collapse of the outer shell during the drying process in the TEM experiment.

\section{Enzymatic stability studies with pepsin and trypsin}

Figure 2 shows the percentage of remaining insulin after incubation of free insulin solution, Ins-SLNs, and SA- $\mathrm{R}_{8}-$ Ins-SLNs in simulated gastric fluid (SGF) with pepsin. Insulin was degraded rapidly by SGF with pepsin within 1 hour. Compared to the free insulin solution, about $45.6 \%$ and $54.7 \%$ of insulin remained at 1 hour incubation of Ins-

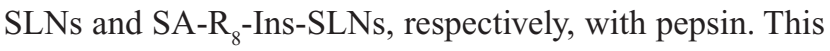
result proved that the SLNs had a certain effect in protecting insulin from degradation in SGF with pepsin. Moreover, the statistical analysis demonstrated that the protective effects of Ins-SLNs were not statistically different $(P>0.05)$ than those of SA-R $\mathrm{R}_{8}$-Ins-SLNs at each time point, suggesting that

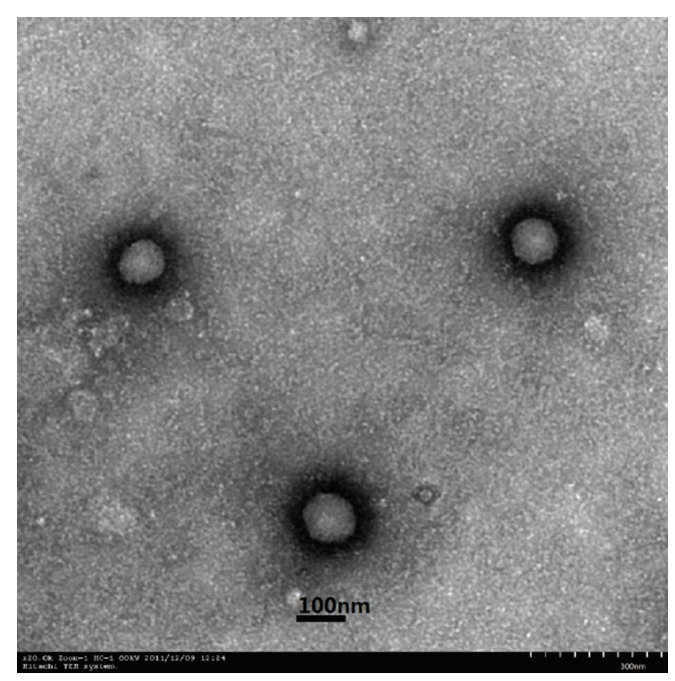

Figure I Transmission electron microscopic (TEM) images of SA-R ${ }_{8}$-Ins-SLNs. Abbreviation: $S A-R_{8}$-Ins-SLNs, insulin solid lipid nanoparticles modified with stearic acid-octaarginine. 


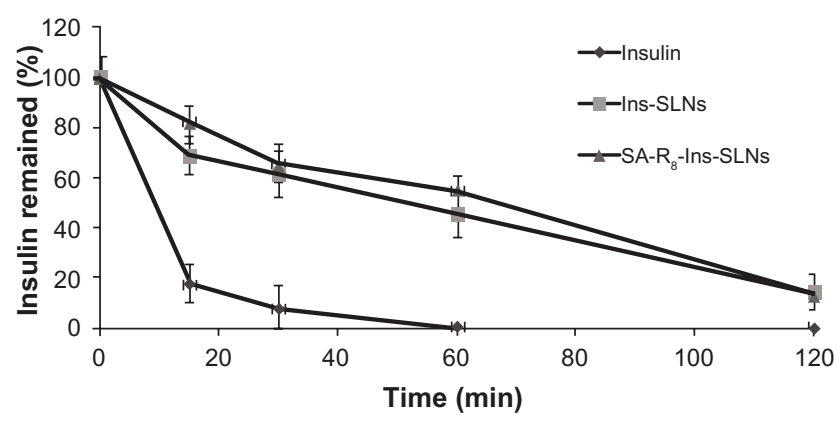

Figure $\mathbf{2}$ The remaining ratio of insulin after the incubation in SGF with pepsin. Abbreviations: Ins-SLNs, insulin solid lipid nanoparticles; $S A-R_{8}-I n s-S L N s$, insulin solid lipid nanoparticles modified with stearic acid-octaarginine; SGF, simulated gastric fluid.

the lipid matrix of the SLNs had an advantage in protecting insulin from enzymatic degradation. This result has been confirmed by several researchers. ${ }^{6,19}$

Figure 3 shows the percentage of remaining insulin after incubation with simulated intestinal fluid in the presence of trypsin, a common intestinal protease. The free insulin was degraded more slowly by trypsin than by pepsin, and only $6.4 \%$ of insulin remained at 4 hours. About $11.6 \%$ of insulin was recovered after 4 hours incubation of Ins-SLNs with trypsin, and about $25.7 \%$ of insulin remained in SA-R - -InsSLNs at 4 hours incubation with trypsin. This finding also suggested that the SLNs and SA- $\mathrm{R}_{8}$ had a synergistic effect in protecting insulin from the gastrointestinal enzymes.

It has been reported that trypsin cleaves insulin at the B22-Arg residue, which is essential for its activity, ${ }^{20}$ while pepsin rapidly cleaves the B24-/B25-Phe and B26-Tyr residues $^{21}$ of insulin. Therefore, $\mathrm{SA}-\mathrm{R}_{8}$ might protect insulin from degradation by the mechanism of competition with trypsin in simulated intestinal fluid, but it had no effect on the degradation activity of pepsin in SGF, due to the fact

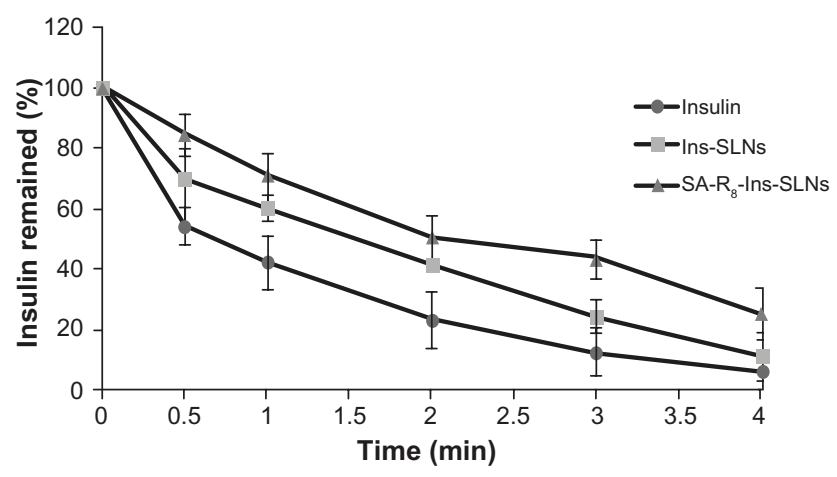

Figure 3 Remaining ratio of insulin after incubation in SIF with trypsin. Abbreviations: Ins-SLNs, insulin solid lipid nanoparticles; $S A-R_{8}-$ Ins-SLNs, insulin solid lipid nanoparticles modified with stearic acid-octaarginine; SIF, simulated intestinal fluid.
Table 2 Apparent permeability coefficient $\left(P_{\text {app }}\right)$ and absorption enhancement ratio $(R)$ for insulin across Caco- 2 cell monolayers

\begin{tabular}{|c|c|c|}
\hline Sample & $P_{\text {app }}\left(\times 10^{-6} \mathrm{~cm} / \mathrm{s}\right)^{*}$ & $\mathbf{R}$ \\
\hline Insulin solution & $0.52 \pm 0.09$ & - \\
\hline Ins-SLNs & $1.17 \pm 0.14$ & 2.25 \\
\hline$S A-R_{8}-I n s-S L N s$ & $9.59 \pm 0.10$ & 18.44 \\
\hline
\end{tabular}

Notes: $\mathrm{n}=6$. *Data were expressed as mean \pm SD of three experiments. Abbreviations: Ins-SLNs, insulin solid lipid nanoparticles; SA- $\mathrm{R}_{8}-$ Ins-SLNs, insulin solid lipid nanoparticles modified with stearic acid-octaarginine; SD, standard deviation.

that $R_{8}$ does not possess the same amino residues cleaved by pepsin. $^{22}$

\section{Transport experiments in the Caco-2 cell}

Table 2 summarizes the $\mathrm{P}_{\text {app }}$ and $R$ values obtained from transport across the Caco-2 monolayer in the presence of insulin, Ins-SLNs, and SA-R - Ins-SLNs. Compared to the

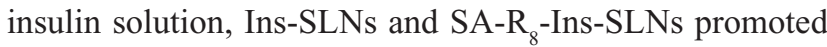
Caco-2 cell internalization by 2.25 and 18.44 times, respectively. This indicated that insulin entrapped in a SLN carrier could pass through the Caco-2 monolayer more efficiently than insulin on its own; moreover, the presence of SA- $\mathrm{R}_{8}$ significantly enhanced transportation efficiency. This result is in agreement with previous reports ${ }^{13,20}$ that $R_{8}$ increased the absorption of insulin.

\section{In vivo hypoglycemic effect}

The glucose response profiles following the duodenal administration of the three samples to streptozocindiabetic rats at a dose of $25 \mathrm{IU} / \mathrm{kg}$ are shown in Figure 4.

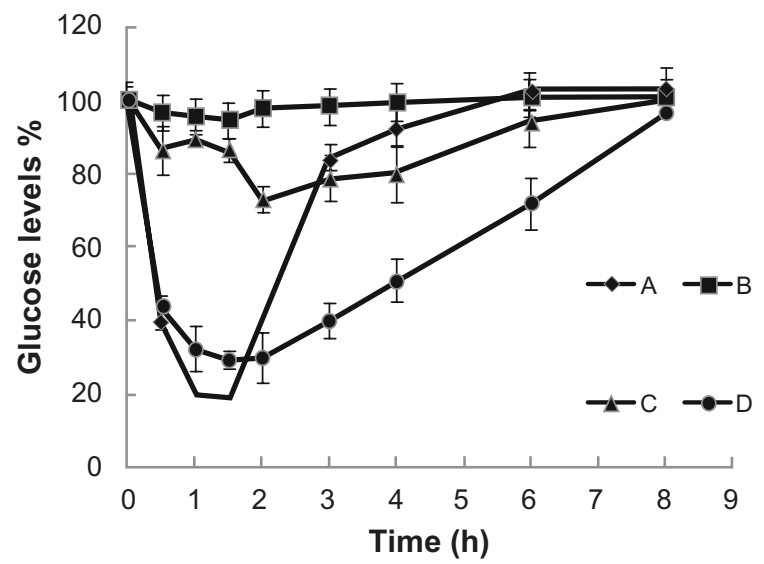

Figure 4 Blood glucose levels changes after subcutaneous or duodenal administration to STZ-diabetic rats. (A) subcutaneous administration of insulin (2 IU/kg); (B) insulin standard solution (25 IU/kg); (C) duodenum administration of Ins-SLNs (25 IU/kg); (D) SA-R - -Ins-SLNs (25 IU/kg).

Abbreviations: Ins-SLNs, insulin solid lipid nanoparticles; $S A-R_{8}-I n s-S L N s$, insulin solid lipid nanoparticles modified with stearic acid-octaarginine; STZ, streptozocin. 
Table 3 The relative pharmacological availability of both SLNs

\begin{tabular}{|c|c|c|}
\hline Sample & AAC (\%) & PA (\%) \\
\hline Insulin solution (25 IU/kg) & $10.05 \pm 4.32$ & $0.42 \pm 0.72$ \\
\hline $\begin{array}{l}\text { Subcutaneous administration } \\
\text { of insulin ( } 2 \mathrm{IU} / \mathrm{kg})\end{array}$ & $189.75 \pm 5.79$ & 100 \\
\hline Ins-SLNs (25 IU/kg) & $100.70 \pm 5.08$ & $4.28 \pm 0.84$ \\
\hline SA-R ${ }_{8}-$ Ins-SLNs (25 IU/kg) & $328.78 \pm 4.76$ & $13.86 \pm 0.79$ \\
\hline
\end{tabular}

Abbreviations: AAC, area above the glucose reduction-time curve; Ins-SLNs, insulin solid lipid nanoparticles; PA, pharmacological availability; SA- $\mathrm{R}_{8}-$ Ins-SLNs, insulin solid lipid nanoparticles modified with stearic acid-octaarginine.

As expected, the insulin standard solution, as a control, showed no hypoglycemic effect. The Ins-SLN group had a certain hypoglycemic effect, with a maximum blood glucose lowering of $73.2 \%$ at 2 hours, while the SA-R - Ins-SLN group achieved a significant hypoglycemic effect, with a maximum blood glucose lowering of $29.7 \%$ at 1.5 hours.

This study was in agreement with previous findings that $\mathrm{R}_{8}$ is a useful tool for delivering macromolecules across cell membranes, as reported by various researchers. ${ }^{20}$ Table 3 lists the pharmacological availability (PA\%) of the three samples administered to the duodenum of diabetic rats. PA\% was calculated relative to the subcutaneous injection of $2 \mathrm{IU}$ insulin $/ \mathrm{kg}$. The PA\% obtained following duodenal

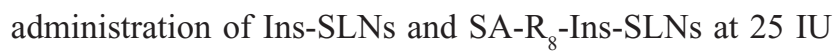
insulin $/ \mathrm{kg}$ was $4.28 \%$ and $13.86 \%$, respectively.

As shown in Table 3, the PA\% of the SA- $\mathrm{R}_{8}$-Ins-SLNs was three times higher than that of Ins-SLNs, with a more apparent hypoglycemic effect (Figure 4). In addition, $\mathrm{SA}_{-} \mathrm{R}_{8}-$ Ins-SLNs promoted internalization of insulin in Caco-2 cells 8.19 times more than Ins-SLNs. This could have been caused by the partial degradation of SA- $\mathrm{R}_{8}$ and insulin in the harsh environment of the gastrointestinal tract. Thus, polymer coating of SA- $\mathrm{R}_{8}$-Ins-SLNs by chitosan, alginate, and hyaluronic acid might be a useful strategy. Further research is in progress in this area.

\section{Conclusion}

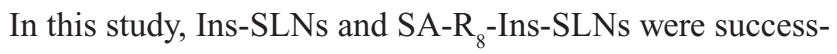
fully prepared by the spontaneous emulsion solvent diffusion method. They were characterized by a spherical morphology, uniform size, positive zeta potentials, and high insulin entrapment efficiency. The typical cell penetration peptide SA- $\mathrm{R}_{8}$ was efficiently absorbed into the $\mathrm{SLN}_{\mathrm{S}}$. Consequently, a dramatic change in zeta potential value between Ins-SLNs $(-32 \mathrm{mV})$ and SA-R - Ins-SLNs $(30 \mathrm{mV})$ was observed, confirming the binding interactions occurring between $\mathrm{SA}_{8} \mathrm{R}_{8}$ with dense positive charges and the negatively charged insulin, soybean phospholipids, and stearic acid.
Insulin was partially protected from gastrointestinal enzymes by incorporation into SLNs and $S A-R_{8}$. SA-R Ins-SLNs increased the uptake of the drug in the Caco-2 cell monolayer, which yielded a more potent hypoglycemic effect following duodenal administration. In conclusion, Ins-SLNs modified with a $\mathrm{SA}-\mathrm{R}_{8}$ drug were able to increase the stability of insulin, improve the transportation efficiency in Caco-2 cells, and increase the in vivo hypoglycemic effect in diabetic rats. Thus, $\mathrm{SA}-\mathrm{R}_{8}$ and $\mathrm{SLNs}$ could be a promising vehicle for oral delivery of insulin. However, further studies should focus more on the stability of insulin in the harsh gastrointestinal environment and the absorption mechanism of the SA-R $\mathrm{R}_{8}$-Ins-SLNs.

\section{Acknowledgments}

The National Natural Science Foundation of China (No 30901867 and No 30973649) is gratefully acknowledged for financial support. This work was also supported by the Research Fund for the Doctoral Program of Higher Education of China (No 20090096110002) and the Fundamental Research Funds for the Central University (JKQ2011016).

\section{Disclosure}

The authors report no conflicts of interest in this work.

\section{References}

1. Bernkop-Schnurch A, Kast CE, Guggi D. Permeation enhancing polymers in oral delivery of hydrophilic macromolecules: thiomer/ GSH systems. J Control Release. 2003;93(2):95-103.

2. Joshi SR, Parikh RM, Das AK. Insulin - history, biochemistry, physiology and pharmacology. J Assoc Physicians India. 2007; 55 Suppl:19-25.

3. Xiong XY, Li YP, Li ZL, et al. Vesicles from Pluronic/poly(lactic acid) block copolymers as new carriers for oral insulin delivery. $J$ Control Release. 2007;120(1-2):11-17.

4. Müller RH, Keck CM. Challenges and solutions for the delivery of biotech drugs - a review of drug nanocrystal technology and lipid nanoparticles. J Biotechnol. 2004;113(1-3):151-170.

5. Elsayed A, Remawi MA, Qinna N, Farouk A, Badwan A. Formulation and characterization of an oily-based system for oral delivery of insulin. Eur J Pharm Biopharm. 2009;73(2):269-279.

6. Morishita M, Peppas NA. Is the oral route possible for peptide and protein drug delivery? Drug Discov Today. 2006;11(19-20):905-910.

7. Garcia-Fuentes M, Torres D, Alonso MJ. Design of lipid nanoparticles for the oral delivery of hydrophilic macromolecules. Colloids Surf B Biointerfaces. 2002;27:159-168.

8. Lee JY, Bae KH, Kim JS, Nam YS, Park TJ. Intracellular delivery of paclitaxel using oil-free, shell cross-linked HSA - multi-armed PEG nanocapsules. Biomaterials. 2011;32(33):8635-8644.

9. Barnes MP, Shen WC. Disulfide and thioether linked cytochrome c-oligoarginine conjugates in HeLa cells. Int J Pharm. 2009;369(1-2): 79-84.

10. Walrant A, Correia I, Jiao CY, et al. Different membrane behaviour and cellular uptake of three basic arginine-rich peptides. Biochim Biophys Acta. 2011;1808(1):382-393. 
11. Takayama K, Tadokoro A, Pujals S, Nakase I, Giralt E, Futaki S. Novel system to achieve one-pot modification of cargo molecules with oligoarginine vectors for intracellular delivery. Bioconjug Chem. 2009;20(2):249-257.

12. Wender PA, Mitchell DJ, Pattabiraman K, Pelkey ET, Steinman L, Rothbard JB. The design, synthesis, and evaluation of molecules that enable or enhance cellular uptake: peptoid molecular transporters. Proc Natl Acad Sci U S A. 2000;97(24):13003-13008.

13. Kamei N, Morishita M, Eda Y, Ida N, Nishio R, Takayama K. Usefulness of cell-penetrating peptides to improve intestinal insulin absorption. J Control Release. 2008;132(1):21-25.

14. Futaki S, Ohashi W, Suzuki T, et al. Stearylated arginine-rich peptides: a new class of transfection systems. Bioconjug Chem. 2001;12(6): 1005-1011.

15. Katayama S, Hirose H, Takayama K, Nakase I, Futaki S. Acylation of octaarginine: implication to the use of intracellular delivery vectors. J Control Release. 2011;149(1):29-35.

16. Mae M, El Andaloussi S, Lundin P, et al. A stearylated CPP for delivery of splice correcting oligonucleotides using a non-covalent co-incubation strategy. J Control Release. 2009;134(3):221-227.

17. Mao S, Germershaus O, Fischer D, Linn T, Schnepf R, Kissel T. Uptake and transport of PEG-graft-trimethyl-chitosan copolymerinsulin nanocomplexes by epithelial cells. Pharm Res. 2005;22(12): 2058-2068.
18. Nakamura K, Murray RJ, Joseph JI, Peppas NA, Morishita M, Lowman AM. Oral insulin delivery using P(MAA-g-EG) hydrogels: effects of network morphology on insulin delivery characteristics. J Control Release. 2004;95(3):589-599.

19. Zhang L, Song L, Zhang C, Ren Y. Improving intestinal insulin absorption efficiency through coadministration of cell-penetrating peptide and hydroxypropyl- $\beta$-cyclodextrin. Carbohyd Polym. 2012; 87(2):1822-1827.

20. Schilling RJ, Mitra AK. Degradation of insulin by trypsin and alpha-chymotrypsin. Pharm Res. 1991;8(6):721-727.

21. Riemen MW, Pon LA, Carpenter FH. Preparation of semisynthetic insulin analogues from bis(tert-butyloxycarbonyl)-desoctapeptideinsulin phenylhydrazide: importance of the aromatic region B24-B26. Biochemistry. 1983;22(6):1507-1515.

22. Fasano A, Uzzau S. Modulation of intestinal tight junctions by zonula occludens toxin permits enteral administration of insulin and other macromolecules in an animal model. J Clin Invest. 1997;99(6): 1158-1164.
International Journal of Nanomedicine

\section{Publish your work in this journal}

The International Journal of Nanomedicine is an international, peerreviewed journal focusing on the application of nanotechnology in diagnostics, therapeutics, and drug delivery systems throughout the biomedical field. This journal is indexed on PubMed Central,

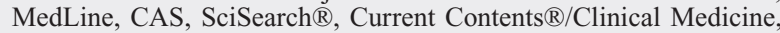

\section{Dovepress}

Journal Citation Reports/Science Edition, EMBase, Scopus and the Elsevier Bibliographic databases. The manuscript management system is completely online and includes a very quick and fair peer-review system, which is all easy to use. Visit http://www.dovepress.com/ testimonials.php to read real quotes from published authors. 